right of the hole in the meso:olon, and this complication will not be seen.

2. Infection of the sutuie Line.-Several French surgeons maintain that romiting beginning a couple of days after the operation may be due to infection of the peritoneum around the suture line. It is quite possible that this may be true, but $I$ have no recollection of seeing it.

3. Adhesion of the Suture Line.-I am persuaded that the commonest cause of persistent and sometimes dangerous vomiting after gastric operations is a sticking together of the anterior and posterior suture lines in the mucosa. Of course, a careless surgcon may actually sew them together, at any rate at a point or two, but apart from this gross technical error it is possible for the cut edges to adhere. If this occurs th: which differs itom that of vicious circle in that no bile is brought up. The cile camnot enter the stomach, and the gastric contents cannot leave it. I first learnt of this danger after performing gastro-jejunostomy for pyloric ulcer.

The patient, a woman, vomited about twice a day for three days, bringing up all she took and much gastric juice besides, but no bile I reopened the abdomen, and found the stomach very large and tense, but the jejunum empty. On opening the jejunum and inserting a finger it was perfectly plain that the anterior and inserting a finger it was perfectly plain that the anterior and
posterior edges at the anastomosis had stuck together. It required
some little force to separate them, but they were not bridged by a stitch. After various other complications she recovered.

A few weeks after a patient with cancer, on whom I had performed a partial resection by the Polya technique, began to vomit the day after operation, and broucht un all she was given and much gastric juice besides, for ther is avs I reopened, and found a huge tens jy ditended stome the also exploration with a finger ingite had adhered tis.itly, but there was sc stitch bridging it. She died.

These two cases brought to my inind other patients at an earlier date, who gave me great anxiety for a few days by persistent vomiting, which then stopped. It is quite likely, of course, that sufficient pressure might be developed in the stomach to force the passage; in fact, in both my cases the stomach was extraordinarily tense. If that happened the condition would be relieved.

In my opinion, then, adherence of the suture line is a not uncommon, and potentially very serious, complication of gastric surgery. Fortunately it is easily prevented. Ever since the experience related above I have inserted a piece of corrugated rubber dam, a little larger than a postage stamp, within the lumen of the anastomosis, fixing it by a fine catgut stitch to the mucosa of the stomach so as to overlap the suture line and point the way to the efferent loop of jejunum. I have had no trouble with vomiting after stomach operations sinco adopting this device. If it be objected that one has to take the clamps off before completing the anterior suture line, in order to insert the piece of dam, the reply is that that ought to be done in any case to make certain that there is no bleeding.

Intestinal anastomoses can be made safe and pervious by the same device.

\section{THE ETIOLOGY AND TREATMENT OF PSORIASIS.} BY

\section{J. H. HEANEY, M.D.}

THE baffling nature of the cause of psoriasis is illustrated by the fact that one dermatologist has even called in the aid of embryology to show an analogy with the scales of lizards. He suggests that psoriasis is a "hark back" defence against cold, since it occurs almost invariably on the elbows and knees, which are the most thinly protected.

The most obvious proposition is that psoriasis is a pal:asitio disease, but there is no cultural or microscopic confirmation of this. The circumstantial evidence consists in (1) well authenticated cases of direct infection; (2) the marginal method of extension; (3) an observed connexion with osteoarthritis; (4) the fact that psoriasis resembles rheumatism in seasonal incidence and tendency to recurrence; and (5) the curative action of parasiticidal medicaments.

There can be no cloubt that poison attacks the skin by way of the blood stream, just as it appears certain that seborrhoea, in the beginning at least, is a more or less widely spread local infection connected with the bottle bacillus, and later associated with certain cocci. Confirmation of this is afforded by the sudden outbursts of activity in old-standing cases, by the symmetrical distribution of the lesions in acute cases, and by acute forms accompanied by asthma and headache, the last named suggesting sensitization. The evidence adduced in favour of other causes is so unconvincing that it might almost be cited in favour of the parasitic theory.

It has been suggested that psoriasis is a stage of seborrhoeic dermatitis, and tho case of a patient, now aged 50, who has bcen under observation for some years will illustrate what is meant. The disease began as a profuse dandruff in early youth; later greasy scaly patches appeared, and still later dry patches on the scalp, followed by dry "spots" here and there on the body. Dry patches subsequently developed on the elbows and lumbar region, and still later extensive dry scaly patches on both shins. This, with two acute attacks of "spots" on the face and hands, extended over the age period 14 to 50 . There is now baldness of the seborrhueic character and very little dandruff, a psoriatic patch on the lumbar region, some involvement over the elbows and shins, and a few patches more truly seborrhoeic in type here and there on the body.

Beginning on the head and extending downwards, this is a classical case of seburrhoea, but if the history and the uppermost lesions are ignored there remains a typical psoriasis of the back, elbows, and shins, extending upwards or not at all. Some say that the two diseases may coexist. At first sight the view that psoriasis is a stage of seborrhoea appears temptingly obvious. What more likely than that a seborrhoeic dermatitis is the forerunner of psoriasis, sometimes in the same subject, as possibly in the case described, or that a seborrhoeic ancestor may have descendants with bald heads and a typical psoriasis beginning below? It is not disputed that a tendency to baldness is hereditary, and it is generally conceded that there is a predisposing disposition to psoriasis.

If it be taken for granted, then, that one of the factors in seborrhoea becomes "fixed" in the blood, and later may cause a psoriasis, which is it? The vegetable parasites to which the bottle bacillus belongs do not usually, if ever, set up blood states, but the cocci do. Moreover, a vaccine of the bottlo bacilli has no effect on psoriasis. On the other hand, chrysarobin has a special lethal effert on vegetable parasites and an almost specific action on psoriasis. Used locally in various strengths it is stirinulant or irritant; it may stimulate growth of hai or cause a dermatitis. It has a special action on primitive : egetable organisms, and kills the spores of ringworm. iwo local actions stand out therapeutically, the stimulant and tho parasiticidal. Recently a patient with chronic psoriasis came under observation. The eruption was almost universal, and the irritation and fall of scales were such that it was feared chrysarobin might precipitate a pityriasis rubra. No treatment had any effect until chrysarobin was prescribed. It is difficult to think of a stimulant effect here. There remains the parasiticidal action and the further possible effect of absorption into the blood stream.

Whitla wrote forty years ago that chrysa obir ointment rubbed into one side of the body alone cured rhe lesions on the opposite side; such an event could only happen through the agency of the blood. How does chrysarobin act in the blood-directly, as salvarsan does, or indirectly? If indirectly, it is possible that, since chrysarobin taken by the mouth induces a rise of temperature, it might give. rise to a copula and complement of its own. The complement, not being specific, might unite with the copula of the disease-another instance of complement deviation-and in that way, or partly in that way, might effect a curo. This would presume a previous deficiency of complement in the blood. Apart from psoriasis, if it were found that chrysarobin was a ready and efficient means of producing complement, its use might become greatly extended.

On the other hand, chrysarobin may act directly on the "poison" both in the blood and in the skin lesions. It is found that the typically seborrhoeic lesions respond better to mercury and tar, but that the drier and more 
chronic ones require chrysarobin. This would point to the presence of a particularly obstinate descendant of the bottle bacillus in these lesions, and to the predominance of the bottle bacillus itself and the special cocci in the more seborrhoeic lesions. In conditions due to the bottle bacillus, such as dandruff, nothing answers better than chrysarobin in weak strength, but where there is a combination of the bacilli and cocci, as in the scaly, greasy forms, chrysarobin shows only irritative properties and does harm, mercury and tar being much more efficient.

For use in the case described above a 0.2 per cent. colloidal solution of chrysarobin was made by Messrs. Crookes with a view to intravenous use; it is not stable in strong solutions at present. Quick progress in so chronic a case is not to be expected, but the results are far better than any hitherto obtained. A daily dose of 30 drops in water one hour after dinner is being given by the mouth, and the solution is well rubbed into the lesions night and morning with a pledget of cotton-wool. The scales are not previously removed; they seem to become partly absorbed where healing is occurring. This solution is the best I have yet tried for dandruff, and seems to promise a permanent cure after short use. It does not discolour the hair, but rather gives it a glint.

It might be concluded, then, on observed facts and the result of treatment-principally the differing actions of chrysarobin-that seborrhoea is the forerunner of psoriasis. Just as we find a gouty joint in the thin, anaemic descendant of a ruddy and gouty ancestor, so psoriasis may occur in the classically accepted form without concomitant sign of its seborrhoeic ancestor. What has been said about treatment was in the first place intended to clarify the etiology, but seems of some importance in itself. Thus the suggested intravenous injections in properly calculated doses might effect a permanent cure of even the most inveterate psoriasis.

It may be added that in the case of seborrhoea recorded acute gout developed at the age of 36 , but the two diseases showed no other common factor except the general good health of the subject.

\section{DIRECT OBSERVATION OF THE CIRCULATION IN THE LIVING IVER.}

BY

JAMES M. McQUEEN, M.B. Сh.B., B.Sc., HALESOWEN, WORCESTERSHIRE.

Using one-quarter to half sized toads (pithed) I have been able, by laying a lobe of the liver on a sheet of glass on the microscope stage, to observe the circulation of the blood in the liver sinusoids and branches of the hepatic vein, under a low power. No cover-glass is required, and no solution of any sort is put in contact with the liver. The liver cells are clearly seen and the course of the blood flowing in the sinusoids (capillaries) can be accurately followed.

The liver circulation is now open to direct observation. Some years ago Leonard Hill greatly extended the microscopic study of the living tissue by his observation of the circulation in the glomerulus of the kidney, and the principle guiding me was the belief that organs apparently too thick for direct observation under the microscope in the adult might not prove so if the observation was made at an earlier stage of growth.

I have noted several times that a pulse may be apparent in the liver sinusoids and in branches of the hepatic veins. It appears to be a pulse of arrest of blood current, due to the contraction of the auricle. It will be easily understood that the blood flows from the liver through auricle into ventricle during diastole of the auricle and ventricle, and when the auricle contracte there is a retardation of the blood current in the liver sinusoids and hepatic veins.

Hence the description given by Sir James Mackenzie in his treatise on the heart is confirmed in great part. If the auricle is able to contract, the pulsation in the liver sinusoids and hepatic veins is auricular in timethat is, the $a$ wave of the liver pulse is more or less synchronous with the $a$ wave in the jugular pulse. If the auricle fibrillates and no proper contraction occurs it acts as a passive receiving chamber, and the systole of the ventricle becomes the retarding agent on the circulation in the liver sinusoids and hepatic veins. Of course this retardation of blood current would be propagated to some extent to the portal vein and to the hepatic artery. Hence the pulse described in the portal vein must have, as one of its factors of origin, this retardation.

Mackenzie believed at one time that the auricle would have to be hypertrophied to produce an auricular form of liver pulse. He also believed an auricular form of liver pulse was a sign of tricuspid stenosis. Latterly he, as stated in his treatise, found cases of auricular liver pulse with no stenosis of the tricuspid valves. Drs. Turnbull and Wiel described such a case.

It will be seen from the foregoing observations that an auricular form of pulse must exist in the liver because the pulse is created in great part simply by retardation and arrest of the blood flow out of the liver. On systole of the auricle the liver swells as the blood flow is arrested because blood is continuously coming to it by the hepatic artery and the portal vein. The demonstration of that pulse is a matter of technique. It would be easier to demonstrate with swelling of the liver sinusoids in cardiac disease and enlargement of the organ. It would not require any hypertrophy of the auricle or tricuspid stenosis to be present. The auricle not only distends the liver -it dams the outflow. Of course, regurgitation of blood into the hepatic veins would add to some extent to the distension of the liver.

It is obvious that the shock of retardation of blood flow falls in great part on the liver cells. This shock is greatly increased when the auricle is practically non-contracting and the ventricle in its systole regurgitates blood through the auricle to the inferior vena cava. The blood pressure in the liver sinusoids must rise, and pulsation with increased capillary pressure is always damaging to cell life. It is apparent in bedsores, where the capillary pressure in the area of contact with the bed is raised by the compression of the venous outlets towards the value of the pressure in the entering arteries. Increased pressure in the capillaries with pulsating shock is disintegrative of cell life.

In the cardiac liver Sims Woodhead notes (Practical Pathology, p. 253):

" that the central or hepatic vein is considerably dilated and the lobules are distinguished more readily than in the norm?! liver. The apillaries leading to this central opening are also dilaic. The capillaries leading to this central opening are also dilared. The liver cells between the dilated capillaries are atrophied, etc. neighbourhood of the central vein."

It will be noted from this description that the pathology follows physiological findings. The pulse of retardation (auricular form normasly present) when exaggerated falls first on the hepatic veins and the liver sinusoids that feed them. Similarly with the ventricular form of pulse of retardation.

But the liver pulse differs from any other form of pulse in that it has three components:

A. The retardation of blood flow by the shuttering off, as it were, of the blood stream through contraction of the auricle. B. Where the auricle is passive as a shuttering off agent, the ventricular systole acts similarly. In both $A$ and $B$ some blood may be driven back into the hepatic veins, but more in the case of $\mathbf{B}$.

C. While $\mathbf{A}$ or $\mathbf{B}$ is in operation the liver swells through the entry of fresh blood by the portal vein and hepatic artery.

Consequently the fall in the liver pulse tracing is due to blood flowing unimpeded out of the liver.

This method of direct microscopical investigation of the liver will lead to further results. The pressure in the liver sinusoids will be measured and light thrown on the problem of the formation of liver lymph. The reactions, if any, of liver sinusoids (capillaries) to various stimuli, etc., are now open to investigation. The question of nerve stimulation and its effects on the liver will no doubt in time be submitted to direct visual examination; and perhaps in the future micro-chemical reactions in the living animal may reveal something of the working of the liver.

I am indebted to the Medical Research Council for the loan of apparatus. 